\title{
Vorhofflimmern
}

\section{Sturzrisiko bei Antikoagulation} überschätzt

Ein erhöhtes Sturzrisiko ist noch immer der häufigste Grund, auf eine orale Antikoagulation bei Vorhofflimmern zu verzichten. Immerhin wird nur noch jedem achten geeigneten Patienten ein Antikoagulans vorenthalten.

$\mathrm{V}$ or Einführung der neuen oralen Antikoagulanzien (NOAK) hatte nur etwa jeder zweite Patient mit Vorhofflimmern eine orale Antikoagulation erhalten. Darauf deutet eine Studie am Uniklinikum Göttingen hin, die PD Maija Djukic vom Institut für Neuropathologie des Klinikums vorstellte. Bei 52\% der Patienten ohne Antikoagulation hätten Ärzte eine erhöhte Sturzgefahr als Grund für den Verzicht auf die Therapie genannt, so Djukic auf der Jahrestagung der Deutschen Gesellschaft für Geriatrie (DGG). In einer aktuellen Analyse in der NOAK-Ära bei 431 Patienten mit Vorhofflimmern waren hingegen nur noch $12 \%$ der Patienten ohne orale Antikoagulation, und bei nur einem Drittel begründeten dies die Ärzte mit einer hohen Sturzgefahr, 22\% nannten ein erhöhtes Blutungsrisiko.

\section{Sturzrisiken werden überbewertet}

Möglicherweise ist aber auch dieser Anteil der unbehandelten Patienten noch zu hoch. Die Neurologin erinnerte an die aktuelle Leitlinie zur SchlaganfallSekundärprävention. Danach ist das Sturzrisiko per se kein Grund für den Verzicht auf die orale Antikoagulation bei Vorhofflimmern. „Sturzrisiken werden meist überbewertet", sagte die Expertin. Nur eine große Studie habe einen klaren Zusammenhang zwischen Sturzrisiko und intrakraniellen Blutungen belegen können. In dieser US-Untersuchung aus dem Jahr 2005 sei zwar die Inzidenz solcher Blutungen mit 2,8\% versus $1,1 \%$ bei Sturzpatienten deutlich höher gewesen, in einer Subgruppenanalyse zeigte sich bei einem $\mathrm{CHADS}_{2}$ Score über zwei Punkten aber ein klarer Nutzen: Die Zahl der verhinderten ischämischen Schlaganfälle war unter oraler Antikoagulation weit größer als die der Hirnblutungen.

Zur Risiko-Nutzen-Abwägung muss natürlich auch die Sturzhäufigkeit beachtet werden. Bei zwei bis fünf Stürzen in der Woche würde er eine orale Antikoagulation infrage stellen, nicht aber bei zwei bis fünf Stürzen im Monat, sagte Prof. Andreas Jacobs vom JohanniterKrankenhaus in Bonn.

Bevor Ärzte jedoch therapeutische Konsequenzen aus dem Sturzrisiko zögen, sollten sie versuchen, dieses Risiko zu senken, so der Neurogeriater. Dazu gehöre die Blutdruckkontrolle und Überprüfung der antihypertensiven Therapie, um Hypotonien $\mathrm{zu}$ vermeiden, sowie eine Untersuchung der Sehfähigkeit. Ferner sollte geprüft werden, ob die Patienten weiterhin psychotrope Medikamente benötigen oder bestimmte Arzneikombinationen das Sturzrisiko erhöhten.

\section{Geringeres Blutungsrisiko unter NOAK}

Zur besseren Verbreitung der Antikoagulation bei Vorhofflimmern dürfte auch das geringere Blutungsrisiko unter den NOAK beigetragen haben. Jacobs erinnerte an die im Vergleich zu Vitamin-K-Antagonisten (VKA) um etwa $50 \%$ reduzierte Gefahr für intrazerebrale Hämorrhagien. Nachteile der NOAK seien die teilweise recht komplexen Dosie- rungsschemata in Abhängigkeit von $\mathrm{Al}$ ter, Körpergewicht und Nierenfunktion sowie die - mit Ausnahme von Dabigatran - bislang fehlenden Antidots. Andererseits seien die NOAKs in den großen Zulassungsstudien auch bei rund 20.000 Patienten über 75 Jahre und 8.000 Patienten über 80 Jahre geprüft worden. Dabei habe sich stets ein günstiges RisikoNutzen-Profil ergeben. Allerdings sei es wünschenswert, weitere Studien bei älteren multimorbiden Patienten vorzunehmen, für diese Gruppe sei die Datenbasis noch etwas dünn, so Jacobs.

Kommt es jedoch zu einer Hirnblutung, ist die Prognose auch unter VKA sehr schlecht. Daran erinnerte Prof. Roland Nau, Chefarzt des geriatrischen Zentrums am Evangelischen Krankenhaus Göttingen-Weende. Nau verwies auf eine Registeranalyse von 141 Patienten mit Hirnblutungen unter VKA. Trotz rascher Gerinnungsnormalisierung mit Vitamin K und ProthrombinKomplex-Konzentrat (PCC) starben $42 \%$ der Betroffenen noch im Krankenhaus. Bei knapp der Hälfte sei es zu einer signifikanten Hämatomexpansion gekommen. Der Experte mahnte, dass bei einer Hirnblutung unter VKA eine Behandlung mit Vitamin $\mathrm{K}$ nicht genüge, es müsse auf jeden Fall zusätzlich PCC verwendet werden, um die Gerinnung zu normalisieren.

Thomas Müller

29. Jahreskongress der Deutschen Gesellschaft für Geriatrie (DGG), 28.-30. 9., Frankfurt a. Main. Symposium S113: Update Schlaganfall im Alter. 